\title{
Mastery quizzes on the Web: Results from a Web-based introductory psychology course
}

\author{
WILLIAM S. MAKI and RUTH H. MAKI \\ Texas Tech University, Lubbock, Texas
}

\begin{abstract}
Quizzes on the Web (QUEB) is a system for delivery of multiple-choice quizzes over the World Wide Web implemented as a set of Perl scripts. Items are contained in a text file and are randomly selected and ordered, and rich feedback is provided to the student. QUEB is in its fifth year of use as a mastery quiz system in an on-line psychology course. QUEB is highly rated by students, and its use is correlated with performance on examinations.
\end{abstract}

In just a few years, the World-Wide Web has become a venue for both on-campus instruction and distance learning. Web-based instruction has progressed from complementing traditional courses with static text-based pages to integrating interactive components such as interactive quizzes and laboratory demonstrations and exercises. The focus of this paper is on the use of interactive quizzes. Specifically, we describe and evaluate a system that we have developed and refined during four years of experience with a Web-based introductory psychology course (R. H. Maki, W. S. Maki, Patterson, \& Whittaker, 2000; W. S. Maki \& R. H. Maki, 1997).

At the time we began development of our on-line course, there were few Web-based quiz systems available, and those that we were aware of did not suit our purposes. A recently published example of such a system is "Quiz-oMatic" (White \& Hammer, 2000). In systems like Quizo-Matic, an instructor logs onto a Web site, types questions and answers into an HTML form, and the tool returns an HTML page. The instructor then downloads the page for deployment to another Web server. In our view, the main problem with this type of system is that the quiz produced by the tool is static-that is, each time the quiz page is accessed, the same quiz items are presented in the same order. Although using such a tool is fairly easy, a substantial amount of time would be spent having to create a mastery system. In a mastery quiz system, students take multiple quizzes covering the same topics but with different questions appearing in each quiz that are drawn from a large pool of items and presented in different orders.

In response, we developed QUEB (Quizzes on the Web). QUEB has been in use each semester since September 1996 in an on-line version of Introductory Psychology taught at two different public universities (North

Support for development and evaluation of the Web course and QUEB was provided by National Science Foundation Grant DUE 9752349 to R.H.M. and W.S.M. Correspondence concerning this article should be addressed to either W. S. Maki or R. H. Maki, Department of Psychology, Texas Tech University, Lubbock, TX 79409-2051 (email: bill.maki@ttu.edu or ruth.maki@ttu.edu).
Dakota State University and Texas Tech University). In the present paper, we describe QUEB and present some data on its effectiveness. To anticipate, we will conclude that (1) mastery quizzes control students' on-line study behaviors when such quizzes carry course credit (Taraban, Maki, \& Rynearson, 1999), (2) students consistently rate the QUEB-based mastery quiz system very highly, and (3) performance on mastery quizzes is correlated with performance on examinations.

\section{METHOD}

\section{Participants}

Participants were enrolled in General Psychology at Texas Tech University during two semesters (Fall and Spring) during each of two successive academic years (1998-1999 and 1999-2000). Initial enrollments totaled $343 ; 32$ students dropped the course, leaving records for 311 students for use in this study. The actual number of student records included depended on the number of complete records for each dependent measure.

\section{Materials}

QUEB is a collection of common gateway interface (CGI) scripts written in Perl. ${ }^{1}$ The system is hosted by an Apache Web server running under the Unix operating system (e.g., Laurie \& Laurie, 1999). (However, QUEB can be exported to other systems supporting Perl and CGI.) The main features of QUEB are listed in Table 1.

Initial setup entails declaring paths pointing to the directories that contain QUEB scripts and files of questions. Only a few other parameters need to be specif ied, notably the number of items per quiz (we used 15) and the criterion for passing (we used $80 \%$ correct). After initialization, the instructor needs to perform three tasks in order to implement QUEB. (1) Using any standard text editor, the instructor must prepare a file of multiple-choice quiz questions. Each question must contain a stem, specif ication of the correct alternative, the location of the associated topic (e.g., page numbers in the textbook), and a variable number of alternatives. Each alternative may be followed by a rejoinder (a brief explanation of why the alternative is correct or not). A separate file needs to be created for each unit of instruction (e.g., a chapter), but the number of questions per file is unlimited. (2) The instructor must prepare a file that contains student usernames and passwords and initialize database files (in plain text format at present) that will contain records of student quiz histories. (3) Standard Web (i.e., HTML) pages containing forms with which to specify the chapter number must be prepared and the students' usernames and passwords must be collected. 
Table 1

Quiz System Features

\section{QUEB Features}

Importing questions and alternatives from text files Random selection of a subset of questions from a large item pool Random ordering of items on the quiz

Random ordering of alternatives

Feedback with explanation of why an alternative is correct or incorrect Historical record of each student's questions attempted and answers given Option to bias item selection toward unseen and/or previously wrong items Detection and prevention of retakes of a quiz

Reports item-by-item quiz results to instructor via e-mail messages Runs on Unix/Linux

\section{Other Desirable Features}

Automatic logging of quiz data in database

Exporting questions and alternatives made in the quiz system to text files Runs on multiple platforms (Windows as well as Unix/Linux)

The software used by the student to gain access to QUEB consists of nothing more than any Web browser, such as Netscape Navigator or Microsoft Internet Explorer. The student uses the browser to request a quiz by submitting an HTML form with his/her username and password. The responding QUEB script consults the quiz history files and determines, for each student, which items have been previously seen, and, of those, which have been answered incorrectly. The script randomly samples from the unseen items. When all items have been seen, the script uses items that have previously been answered incorrectly. Items that have previously been answered correctly are used only if they are necessary to complete the quiz. The script presents the selected items in a random order. Each presented item is a multiple-choice question that contains the stem of the question and a list of alternatives specified for that question. The alternatives are presented with radio buttons to the left of each item so that only one alternative can be selected for each question. A button is inserted at the end of the quiz; selecting that button submits the form thus requesting that the quiz be scored. A string with the question numbers and selected alternatives is then sent to a separate script that scores the student's responses.

Web browsers allow a user to return to recently seen pages. This browser feature presents a problem for on-line quiz systems; after receiving feedback, the student can back up to the page of quiz questions and revise the answers and resubmit the quiz. Any browser-level intervention that can be defeated by the user (such as "cookies") will not be an effective countermeasure. Thus, the quiz memory system in QUEB was developed to prevent students from backing up in order to revise a quiz that has just been scored. Before a quiz is scored, the QUEB scoring script uses the questions and alternatives in the submitted quiz to create a new quiz memory string. The string contains a list of (randomly ordered) question numbers and randomly ordered lists of alternatives for each question. The string is very long; with 15 questions per quiz and four alternatives per question, the result is a string of 75 randomly ordered 1- and 2digit numbers. (The likelihood of an accidental match is extremely small.) If the new string matches the quiz memory string retrieved from the student's quiz memory file, a message is displayed announcing that the scoring attempt is refused. If the strings do not match, the new string is saved in the student's quiz memory file and scoring proceeds.

In addition to scoring the student's responses, the scoring script updates the quiz history files, displays a marked-up version of the quiz to the student, and sends e-mail containing the information about the quiz to the instructor. The marked-up version displayed to the student shows whether each question was answered correctly or incorrectly. Each alternative is redisplayed in the marked-up ver- sion together with the rejoinder for each alternative and the location information (e.g., page number) for that question. At the end of the marked-up quiz, the student is informed of the percentage correct and shown a reminder about the criterion for passing. The e-mail message sent to the instructor contains the percentage correct for that quiz, the student's quiz history (items answered correctly, items answered incorrectly, and items not yet seen), and an item analysis (student response and correct answer for each item in the quiz).

The questions used in the quizzes were taken, with permission, from the test bank furnished by the publisher of the textbook used in the course. Approximately half the questions were extracted (by an odd-even split-half method) and used for the mastery quizzes. The remaining questions were used to construct in-class examinations.

\section{Procedure}

Four graduate student teaching assistants (TAs) participated as instructors of the on-line course, two during each academic year. Four groups of students (sections) met at different times each semester. Each TA was responsible for two of the sections each semester. Instructors differed in gender, area of specialization, amount of teaching, and other academic experience.

On-line activities were substituted for most class meetings in the Web-based General Psychology course, and completion of the online activities earned course credit (R. H. Maki et al., 2000). The QUEB system was used to support three different learning aids in the course. First, students were required to pass two mastery quizzes each week and could take up to two more for additional credit. Second, course outlines and questions frequently asked (FAQs) by beginning psychology students were posted on the Web; students were encouraged to preview these study materials before studying each chapter, and QUEB was used to present a short "study" quiz (five items) for each chapter. Third, the students worked on weekly homework assignments involving, for example, seeking information on the Web or collecting data in brief on-line experiments; another short quiz (five items) was presented using QUEB at the end of each of these weekly assignments.

Two other course components were not on line. The class met once each week under the direction of the TA to discuss current textbook readings and/or to aggregate and discuss data collected in the weekly homework assignments. Also, students participated in psychological research projects for course credit.

After every three or four chapters (i.e., every three or four weeks), an in-class examination was scheduled. The exam was administered as a paper-and-pencil test but the format was similar to the on-line quizzes; each exam question was multiple choice with four or five alternatives. A total of 31 students did not take the final examination, leaving 280 complete records to be analyzed of the correlates of examination performance.

At the midpoint of the semester, a midterm course evaluation was administered. The questions providing data for this study were of the form: "How useful are X for learning course material?" where $\mathrm{X}$ was the name of the course component being evaluated. The students rated these components using a 5-point rating scale with 5 being most positive. The response alternatives (ordered from high to low) were "very useful," "moderately useful," "slightly useful," "not at all useful," and "they interfere with learning." The analyses included those five components for which ratings were collected during each of the four semesters: mastery quizzes, FAQs, study quizzes, weekly computer assignments, and class meetings. A total of 264 student evaluations were received of which 250 were complete and were used in our analyses.

\section{Results}

Here we will consider three outcomes: student use of the QUEB system, student evaluations of QUEB relative 
to evaluations of other course components and another quiz system (i.e., Quiz-o-Matic), and the relation between various course components and examination performance.

Behavioral effects. The use of QUEB for mastery quizzes was a mandatory part of the course, and the quizzes needed to be passed at a criterion of $80 \%$ correct on or before the weekly due date. Students earned points for passing up to four mastery quizzes each week. We know that this causes weekly quiz-taking behaviors (Taraban et al., 1999) by over $95 \%$ of the students (W. S. Maki \& R. H. Maki, 2000). In contrast, White and Hammer (2000) reported only $56 \%$ of the students using the optional Quiz-o-Matic system. The differing usage rates might depend on the amount of course credit assigned to the quizzes, the nature of the quiz system, or both.

Student evaluations. How did QUEB mastery quizzes compare with Quiz-o-Matic? Surprisingly, the (mandatory) QUEB system was rated higher than was the (optional) Quiz-o-Matic system. A total of $95 \%$ of our students rated the mastery quizzes as moderately or very useful for learning, averaging $4.75(S D=0.55, N=$ 250 ) on our 5 -point scale. White and Hammer (2000) reported that their Quiz-o-Matic system was rated "generally useful," averaging $5.65(S D=1.04, N=161)$ on a 7-point scale. The score of 5.65 on the White and Hammer 7-point scale is equivalent to 4.04 on our 5 -point scale; the 4.75 on our 5 -point scale is equivalent to 6.65 on a 7-point scale. Single-sample $t$ tests showed that the rating of QUEB-based mastery quizzes was significantly higher than the rating of Quiz-o-Matic, either using the statistics from the QUEB sample and converting to the 5 -point scale $[t(249)=20.41, p<.001]$ or using the statistics from the Quiz-o-Matic sample and converting to a 7-point scale $[t(160)=12.20, p<.001]$.

How did QUEB mastery quizzes compare with other course components? Ratings of the QUEB-based mastery quizzes were higher in every semester than were ratings of the other components of the course. Table 2 contains means and standard deviations of the ratings of the mastery quizzes, FAQs, study quizzes, assignments, and class meetings; the data are averaged over all semesters and are also presented separately for each of the four semesters. The ratings were subjected to a $5 \times 4$ mixed analysis of variance that included the five course components (as the within-subjects factor) and four semesters (as the between-groups factor). The overall average course component ratings differed significantly, as shown by the significant main effect of course component $[F(4,984)=$ $\left.90.01, M S_{\mathrm{e}}=0.60, p<.001\right]$. The ratings for mastery quizzes were significantly higher than the average ratings assigned to the other four components $[F(1,246)=$ $\left.119.07, M S_{\mathrm{e}}=0.27, p<.001\right]$. However, the ratings of these other four components varied across semester, as indicated by the significant main effect of semester $\left[F(3,246)=4.93, M S_{\mathrm{e}}=1.37, p<.01\right]$ and by the significant interaction of component and semester $[F(12,984)=$ $\left.7.07, M S_{\mathrm{e}}=0.60, p<.001\right]$. Simple main effects (of semester for each component) showed that the ratings of the mastery quizzes did not vary significantly from semester to semester $(F<1)$. But the average ratings of each of the other four components did vary across semesters $\left[F(3,246)=4.81, M S_{\mathrm{e}}=0.82, p<.01 ; F(3,246)=\right.$ $8.47, M S_{\mathrm{e}}=0.72, p<.001 ; F(3,246)=2.62, M S_{\mathrm{e}}=0.92$, $p<.06$; and $F(3,246)=10.82, M S_{\mathrm{e}}=1.03, p<.001$, for FAQs, study quizzes, assignments, and class meetings, respectively].

Course components and exam performance. We have observed significant correlations between performance on mastery quizzes and performance on in-class examinations. Total points obtained for mastery quizzes, assignments, FAQs, class meetings, and examinations were computed for each student who had complete sets of scores for these measures $(N=280)$. The product-moment correlations among all five measures across all four semesters are shown in Table 3. (Results from individual semesters were comparable.) The correlations in the table show that performances on mastery quizzes and assignments were more highly correlated with examination performance than were points obtained for research participation and class meetings. However, points for mastery quizzes and assignments were also correlated with points for research participation and class meetings. Partial correlations were computed, controlling for research and class. Mastery quiz and assignment performances still correlated with examination performance $[r(276)=.407$, and $r(276)=.425$, respectively, $p \mathrm{~s}<.01]$. A final partial correlation was computed that also controlled for the variance due to assignments; the correlation between mastery quiz performance and examination performance was lower but still significant $[r(275)=.259, p<.01]$.

Table 2

Average Ratings of Class and On-Line Course Components Across Four Semesters

\begin{tabular}{|c|c|c|c|c|c|c|c|c|c|c|}
\hline \multirow[b]{3}{*}{ Measure } & \multicolumn{10}{|c|}{ Semester } \\
\hline & \multicolumn{2}{|c|}{ F98 } & \multicolumn{2}{|c|}{ S99 } & \multicolumn{2}{|c|}{ F99 } & \multicolumn{2}{|c|}{ SO0 } & \multicolumn{2}{|c|}{ Average } \\
\hline & $M$ & $S D$ & $M$ & $S D$ & $M$ & $S D$ & $M$ & $S D$ & $M$ & $S D$ \\
\hline Mastery & 4.69 & 0.65 & 4.67 & 0.65 & 4.80 & 0.47 & 4.78 & 0.47 & 4.75 & 0.55 \\
\hline FAQ & 4.04 & 0.87 & 3.73 & 0.81 & 3.58 & 1.00 & 3.44 & 0.89 & 3.66 & 0.92 \\
\hline Study & 3.76 & 0.95 & 3.62 & 0.89 & 4.32 & 0.81 & 4.06 & 0.80 & 3.98 & 0.89 \\
\hline Assignment & 3.86 & 0.89 & 4.00 & 0.91 & 3.93 & 0.96 & 3.58 & 1.03 & 3.82 & 0.97 \\
\hline Class & 3.84 & 1.05 & 3.60 & 0.96 & 3.63 & 1.05 & 2.91 & 1.01 & 3.44 & 1.07 \\
\hline$N$ & \multicolumn{2}{|c|}{49} & \multicolumn{2}{|c|}{52} & \multicolumn{2}{|c|}{71} & \multicolumn{2}{|c|}{78} & \multicolumn{2}{|c|}{250} \\
\hline
\end{tabular}

Note-Data are mean ratings based on the $N$ s indicated in the last row. Semesters are Fall (F) and Spring (S) from the 1998-1999 and 1999-2000 academic years. 
Table 3

Correlations Between Mastery Quizzes and Other Course Components

\begin{tabular}{llcccc}
\hline & \multicolumn{5}{c}{ Component } \\
\cline { 2 - 6 } \multicolumn{1}{c}{ Component } & Study & Assignments & Research & Class & Exams \\
\hline Mastery quizzes & $.443^{* *}$ & $.566^{* *}$ & $.313^{* *}$ & $.332^{* *}$ & $.428^{* *}$ \\
Study & & $.548^{* *}$ & $.313^{* *}$ & $.433^{* *}$ & $.356^{* *}$ \\
Assignments & & & $.360^{* *}$ & $.42^{* *}$ & $.441^{* *}$ \\
Research & & & & $.404^{* *}$ & .089 \\
Class & & & & & $.153^{*}$ \\
\hline
\end{tabular}

Note $-N=280$ for all correlations. *Significant at the 0.05 level (2-tailed). ***Significant at the .01 level (2-tailed).

\section{DISCUSSION}

In summary, this study of a mastery quiz system deployed over the Web leads us to draw three conclusions. First, when a quiz system is coupled with appropriate contingencies specifying amounts of course credit and deadlines for completion, the vast majority of students use the system. Second, in each of the four semesters reported here, the students rated the mastery quiz system more highly than any other course component and more highly than another quiz system-Quiz-o-Matic, recently reported by White and Hammer (2000). Third, performance on mastery quizzes is correlated with performance on examinations, and the correlation remains even after controlling for the variance due to other course components. Each of these conclusions is considered in more depth in the following discussion.

\section{Use of Web-Based Materials}

We continue to believe that on-line quizzes such as the QUEB must be accompanied by contingencies that will ensure their usefulness; otherwise, students will allocate their time to other academic (or nonacademic) activities and the Web-based materials will go unused. In the courses reported here, mastery quizzes and other on-line activities replaced traditional lectures and allowed students to accumulate considerable amounts of course credit (W. S. Maki \& R. H. Maki, 2000). The technology inherent in the World Wide Web allowed us to record the students' use of and performance on various course components. Thus, we were assured that nearly all of the students did use this system (W. S. Maki \& R. H. Maki, 2000) and that their behaviors were controlled by the contingencies associated with the various course components (Taraban et al., 1999).

Almost all (95\% on average) of our students used QUEB for taking mastery quizzes. But did they use it as we intended, as a diagnosis of their content knowledge? And, if not, what would the implications be for our conclusions?

Web browsers, although relatively simple to use, permit two misuses of QUEB. The first such misuse is permitted by the browser's "back" button that can return the user to the most recent previously seen page. The implication is that students can revise and resubmit previously scored quizzes. This problem is countered in QUEB by the quiz memory system. When a quiz is submitted for scoring, QUEB creates a very long string of numbers from the sequence of questions and alternatives. The quiz is scored only if the string does not match the string in that student's quiz memory. Thus, students in the courses reported here could not take advantage of Web browser features to revise previously scored tests.

The second misuse of quiz systems permitted by Web browsers is the printing of (scored) quizzes. From anecdotes gathered in discussions with students, we know this happens frequently, and we know from these discussions that students use the printed copies to prepare for the in-class examinations. We routinely advise students not to exclusively use printed quizzes as study aids because the examinations do not contain the same questions. But we admit that this use of QUEB was not controlled in this study and thus it may have mediated the observed quiz-examination correlation and student ratings.

\section{Student Evaluation of Mastery Quizzes}

The students not only used the mastery quiz system, but their ratings of the system suggest that they liked it. The stability of the ratings of mastery quizzes (vs. the fluctuations of ratings of other course components) across semesters and instructors is remarkable because of a similar pattern of results noted by R. H. Maki et al. (2000). R. H. Maki et al. observed that students in the Web-based course performed better than did students in the lecture-based version on tests of content knowledge. This difference was shown in both exam performance and in pretest- posttest gains on an independent set of questions. Moreover, these differences were stable across different semesters and different instructors. In contrast, other dependent measures (such as overall course ratings) were influenced by both semester and instructor. It may be only coincidental that student ratings of usefulness of mastery quizzes for learning and content knowledge gains are both stable across semesters and instructors. But it may be that student ratings of mastery quizzes are a reflection of what these quizzes contribute to the amount learned in the Web-based course.

\section{Quizzes, Examinations, and Learning}

It appears that performance on mastery quizzes is correlated with or predictive of performance on in-class examinations. But such correlations are notoriously difficult to interpret. We were able to rule out some general motivational factors (with the partial correlation coefficients controlling for research and class participation), and we showed a residual correlation between mastery quiz and examination performances even when the influences of other quiz-based activities (study quizzes and assignments) were statistically controlled. One possible explanation for the correlation is that students' learning from quizzes is expressed on the examinations. But the correlation between mastery quizzes and examinations still could be due to some other "third" variable. This alternative explanation, however, must be constrained by several results, which we have accumulated in our eval- 
uations of Web-based versus lecture versions of the general psychology course. (1) Examination performances were consistently higher in the Web-based than in the lecture sections of this course (R. H. Maki et al., 2000). (2) Students in the Web-based sections gained more across the semester on an independent test of content knowledge than did students in the lecture sections (R. H. Maki et al., 2000). (3) Students rated the quiz system as useful for learning, as discussed above. (4) Our present results show that the correlation between quiz and exam performance cannot be explained by some general motivational factor or by correlations with other on-line course components. (5) As in the present study (and also in R. H. Maki et al., 2000), the variables most directly related to student learning seem to have been independent of instructor and semester. In order to explain these facts, the "third" variable would have to operate in such a way as to increase both the mean exam performance and the pretest-posttest gains in content knowledge only in the Web sections. The third variable would have to be correlated with both quiz and exam performance and would have to cause high student ratings of quizzes. It would also have to be independent of the effects of instructor and semester. It seems more parsimonious to think of the QUEB-based mastery quizzes as contributing somehow to learning as measured by examination performance.

\section{Recommendations}

Since we began our work on the evaluation of on-line learning, several other quiz systems have been described that have some of the same features present in QUEB (see Table 1). Some of these are stand-alone quiz systems, such as Perception (http://www.qmark.com) and Quia (http://www.quia.com). Other assessment tools are embedded in course management systems that are available commercially, for example, WebCT (http://www. webct.com), Blackboard (http://www.blackboard.com), and TopClass (http://www.wbtsystems.com). We expect that the use of these systems would produce levels of student learning and satisfaction similar to those reported here, to the extent that the systems include the features noted in Table 1 and are coupled with behavioral contingencies that will ensure their use.

One of the commercial learning management systems should be considered for another practical reason. QUEB was originally developed as a research tool. Consequently, it has some features that are peculiar to its history and its developers. For example, in its present form, quiz results are reported to the course instructor by e-mail messages. Data are then extracted from electronic mailbox files by other scripts running locally on a desktop workstation. Automatic filtering of messages into various mailboxes and folders makes the system workable; however, reporting of quiz scores to students is delayed by the off-line intervention. It would be desirable to have the output from the quiz system fed directly to a course grade-book system in such a way as to reduce instructor management load, speed feedback to students, and increase scalability to courses with larger enrollments.

Instructors who enhance a course with on-line quizzes (or who teach a distance learning course) cannot be expected to develop their own quiz systems; neither is it realistic to use a system like QUEB, which was built for research purposes. Using one of the course management systems appears to be an attractive alternative. We have described the features (in Table 1) that we think instructors should seek as they evaluate the various commercial quiz systems. On the basis of the results reported here, we anticipate that the use of systems with these features will lead to high student satisfaction with the quiz system and effective learning.

\section{REFERENCES}

Laurie, B., \& LaUrie, P. (1999). Apache: The definitive guide (2nd ed.). Sebastopol, CA: O'Reilly.

Maki, R. H., Maki, W. S., Patterson, M., \& Whittaker, P. D. (2000). Evaluation of a Web-based introductory psychology course: I. Learning and satisfaction in on-line versus lecture courses. Behavior Research Methods, Instruments, \& Computers, 32, 230-239.

MAKI, W. S., \& MAKI, R. H. (1997). Learning without lectures: A case study. IEEE Computer, 30, 107-108.

MAKI, W. S., \& MAKI, R. H. (2000). Evaluation of a Web-based introductory psychology course: II. Contingency management to increase use of on-line study aids. Behavior Research Methods, Instruments, \& Computers, 32, 240-245.

Schwartz, A. (1998). Tutorial: Perl, a psychologically efficient reformatting language. Behavior Research Methods, Instruments, \& Computers, 30, 605-609.

Stein, L. (1998). Official Guide to Programming with CGI.pm. New York: Wiley.

Taraban, R., Maki, W. S., \& Rynearson, K. (1999). Measuring study time distributions: Implications for designing computer-based courses. Behavior Research Methods, Instruments, \& Computers, 31, 263-269.

Wall, L., Christiansen, T., \& Schwartz, R. L. (1996). Programming Perl (2nd ed.). Sebastopol, CA: O'Reilly.

White, R. J., \& Hammer, C. A. (2000). Quiz-o-Matic: A free Webbased tool for construction of self-scoring on-line quizzes. Behavior Research Methods, Instruments, \& Computers, 32, 250-253.

\section{NOTE}

1. In spite of our positive experiences with QUEB, we admit that QUEB is not an out-of-the-box product. Using QUEB requires some knowledge of Perl (A. Schwartz, 1998), familiarity with computer operating systems, text editing skills, and an understanding of electronic mailbox formats in order to extract student scores from messages. A. Schwartz provided a tutorial on Perl for this journal. Other references used in the development of QUEB include the reference manual by Wall, Christiansen, and R. L. Schwartz (1996) and the CGI.pm Perl library by Stein (1998). The complete source code for QUEB is available at no cost on an "as-is" basis for faculty members who wish to use this web-based quiz system for noncommercial purposes. The scripts can be downloaded from http://classes.ttu.edu:8902/downloads.

(Manuscript received December 19, 2000; accepted for publication March 21, 2001.) 